\title{
Redução do déficit de estatura e a compra de alimentos da agricultura familiar para alimentação escolar no Brasil
}

\author{
Genykléa Silva de Oliveira \\ Clelia de Oliveira Lyra* \\ Angelo Giuseppe Roncalli da Costa Oliveira ${ }^{\star \star *}$ \\ Maria Angela Fernandes Ferreira ${ }^{\star * \star}$
}

\begin{abstract}
O objetivo do artigo é analisar o efeito da compra direta de alimentos da agricultura familiar para alimentação escolar sobre o déficit de estatura em crianças menores de cinco anos, entre 2013 e 2017, no Brasil. 0 método utilizado é o estudo ecológico e longitudinal, em que a coleta de dados ocorreu em diferentes bancos de dados. A análise foi realizada por meio do teste $t$ para amostras independentes, a fim de comparar as médias entre as variáveis déficit de estatura e compras da agricultura familiar. Além disso, uma análise multivariável foi feita por meio da regressão linear múltipla. Verificou-se uma diferença de médias na prevalência do déficit de estatura significativa entre os municípios que compraram menos de $30 \%$ e aqueles que adquiriram $30 \%$ ou mais de alimentos da agricultura familiar para alimentação escolar, com 1,47 pontos de diferença. 0 modelo de regressão linear mostrou que a cada ponto percentual de aumento na compra de alimentos da agricultura familiar para a alimentação escolar pelo município, haverá, em média, uma diminuição 0,55 pontos na prevalência do déficit de estatura, independentemente das demais variáveis. Assim, conclui-se que a compra de alimentos da agricultura familiar para alimentação escolar no Brasil pode contribuir para a redução da prevalência do déficit de estatura em crianças menores de cinco anos.
\end{abstract}

Palavras-chaves: Segurança alimentar e nutricional. Desnutrição infantil. Alimentação escolar. Agricultura.

\footnotetext{
* Universidade Federal do Rio Grande do Norte (UFRN), Natal-RN, Brasil (genyklea@yahoo.com.br; https://orcid.org/00000002-7054-3612).

** Universidade Federal do Rio Grande do Norte (UFRN), Natal-RN, Brasil (clelia_lyra@yahoo.com.br; http://orcid.org/00000002-1474-3812).

${ }^{* \star \star}$ Universidade Federal do Rio Grande do Norte (UFRN), Natal-RN, Brasil (roncalli@terra.com.br; https://orcid.org/00000001-5311-697X).

**** Universidade Federal do Rio Grande do Norte (UFRN), Natal-RN, Brasil (mangelaf50@gmail.com; https://orcid. org/0000-0002-6142-948X).
} 


\section{Introdução}

O déficit do crescimento reflete problemas nutricionais crônicos e que ocorreram por interferência de múltiplos fatores: genéticos, nutricionais, sociais e econômicos. Por isso, a avaliação do estado nutricional de crianças menores de cinco anos é um indicador importante utilizado mundialmente para verificar as condições de saúde e qualidade de vida da população (GARCIA, 2018; AKOMBI et al., 2017; PEREIRA et al., 2017; MONTEIRO et al., 2009).

Para monitorar o déficit de crescimento em crianças menores de cinco anos, são utilizadas, internacionalmente, as curvas de crescimento da Organização Mundial de Saúde (2006), por meio da avaliação dos índices antropométricos, em virtude de serem capazes de detectar os desequilíbrios nutricionais desde quadros de desnutrição até a obesidade (WHO, 2006; SBP, 2009). Em especial para a avaliação da desnutrição, os indicadores antropométricos associados capturam os efeitos não só da insegurança alimentar, mas também, de maneira indireta, da falta de acesso a serviços de saúde e de doenças como diarreia, malária, HIV/Sida e tuberculose (FAO, 2013).

No mundo, a prevalência do déficit de crescimento entre crianças menores de cinco anos está diminuindo: passou de 165,8 milhões em 2012 para 148,9 milhões em 2018 (FAO et al., 2019). Embora represente uma queda de 10,1\% no período de seis anos, não atingiu a redução que era esperada de $20 \%$, ficando assim difícil alcançar um ritmo adequado de decréscimo, a fim de atingir a meta estipulada para 2030, que seria de diminuir pela metade o número de crianças com déficit de crescimento em comparação a 2012 (FAO et al., 2019).

0 declínio relatado anteriormente no déficit de crescimento que ocorreu na maioria dos países da América Latina, inclusive no Brasil, foi em consequência de melhorias nas condições socioeconômicas e no acesso a serviços de saúde, saneamento e educação (PEDRAZA; SALES; MENEZES, 2016; ONIS; BLOSSNER; BORGHI, 2020).

Alguns estudos mostram que os programas voltados para acabar com a fome e promover a segurança alimentar e nutricional (SAN) no Brasil, como Programa Fome Zero (2003) que tinha vários outros programas atrelados, destacando-se o Bolsa Família, teriam contribuído para a redução da desnutrição no país (PEREIRA et al., 2017; MARTINS, 2013).

Outra ação que pode ser citada como importante para a promoção da SAN no Brasil é o Programa Nacional de Alimentação Escolar (PNAE), um dos mais antigos e abrangentes no âmbito da garantia universal da SAN e do Direito Humano à Alimentação Adequada (DHAA), ofertando alimentos aos alunos da educação básica e filantrópica de ensino no país. Este programa foi instituído em 1955, pelo Ministério da Educação (MEC), e atendeu a mais de 40 milhões de estudantes no país em 2015 (PEIXINHO, 2013; FNDE, 2020).

Um marco legal que promoveu avanços fundamentais para o alcance da SAN por meio do PNAE, no Brasil, foi a Lei n. 11.947/2009, na qual, entre outras mudanças apresentadas, destaca-se o incentivo ao desenvolvimento local sustentável, instituindo a obrigatoriedade da compra de no mínimo 30\% de gêneros alimentícios provenientes diretamente da agricultura familiar e do empreendedor familiar rural ou suas organizações, privilegiando os 
assentamentos da reforma agrária, as comunidades tradicionais indígenas e comunidades quilombolas (PEIXINHO, 2013; BRASIL, 2009).

Nesta perspectiva, a questão-alvo do presente estudo é: a aquisição de alimentos da agricultura familiar para a alimentação escolar contribui para redução do déficit de estatura de crianças brasileiras? Logo, a fim de responder a essa pergunta, o objetivo do estudo foi analisar os efeitos da compra direta de alimentos da agricultura familiar para alimentação escolar sobre o déficit de estatura em crianças menores de cinco anos acompanhadas nos serviços de atenção básica à saúde no Brasil, entre 2013 e 2017.

\section{Método}

Trata-se de uma pesquisa com uso de dados secundários e classificada, portanto, como um estudo ecológico e longitudinal do período de 2013 a 2017. A população de estudo foram crianças menores de cinco anos atendidas nos serviços de atenção básica nos 5.570 municípios brasileiros de 2013 a 2017. Neste período foram avaliadas, em média, 4.488.230 crianças nos serviços de atenção básica na faixa etária em questão (BRASIL, 2020). Os dados analisados foram coletados nos bancos de dados do Sistema de Vigilância Alimentar e Nutricional (Sisvan/Ministério da Saúde) (https://sisaps.saude. gov.br/sisvan/), em dezembro de 2019.

A variável desfecho do estudo foi o déficit de estatura em crianças menores de cinco anos, avaliado conforme os indicadores propostos pela Organização Mundial de Saúde (OMS), em que a baixa estatura para idade é classificada com o valor de estatura abaixo de menos 2 desvios-padrão da mediana (abaixo de -2 escores-z) (WHO, 2006).

Portanto, neste estudo, foi considerado "déficit" de estatura a soma do percentual de crianças com estatura baixa e muito baixa para a idade, dados que foram obtidos por meio dos relatórios do Sisvan nos anos do estudo. A escolha da faixa etária deveu-se ao fato de a avaliação do estado nutricional de crianças menores de cinco anos ser um dos indicadores de uso internacional para monitoramento de saúde e nutrição da população (FAO, 2014).

Em relação à variável independente principal, o percentual de recursos utilizados na compra de gêneros alimentícios provenientes diretamente da agricultura familiar e do empreendedor familiar rural ou suas organizações para a alimentação escolar pelos municípios brasileiros foi coletado no site do FNDE (Fundo Nacional de Desenvolvimento da Educação), para o período de 2013 a 2017 (FNDE, 2019). Esta variável foi classificada como "não cumpriu" para os municípios que utilizaram menos de $30 \%$ dos recursos para aquisição de gêneros alimentícios da agricultura familiar e, como "cumpriu”, para aqueles que gastaram $30 \%$ ou mais dos recursos financeiros nesse tipo de compra, conforme disposto na legislação.

As demais variáveis contextuais relativas a condições socioeconômicas foram coletadas nos seguintes bancos de dados: PNUD, IBGE e Ipea, conforme apresentado no Quadro 1 do Anexo. 
O banco de dados foi organizado pela técnica de linkage determinístico a partir do geocódigo do município das variáveis de estudo. Em seguida, realizou-se um levantamento de dados perdidos e atípicos (outliers) por meio da análise descritiva e da distância de Mahalanobis $\left(D^{2}\right)$ com probabilidade de menor que 0,001. Após esta etapa foram excluídas duas variáveis, pois apresentaram mais de $5 \%$ de missing com significância estatística conforme o teste $t$ e a aleatoriedade das perdas foi analisada a partir do teste de Little, obtido com a análise EM (expectation-maximization) (p<0,0001). Ademais, mais cinco municípios foram excluídos por terem mais de $90 \%$ de missing. Também foram eliminados outliers univariados da variável independente principal (10) e multivariados (139) detectados pela medida $D^{2}$ pela média da variável com dado atípico. Assim, as análises foram realizadas em 5.416 municípios (97\%).

Foram utilizados os seguintes softwares para a análise: Microsoft ${ }^{\circledR}$ Exce $^{\circledR} 2013$ para organização dos dados e o IBM ${ }^{\circledR} S P S S^{\circledR}$ versão 26 para a análise estatística dos dados.

Primeiro, foi realizado o teste $t$ para amostras independentes, a fim de comparar as diferenças de médias do período analisado na compra da agricultura familiar e no déficit de estatura estratificados por regiões brasileiras. Nestas análises a média do déficit de estatura nos municípios foi ponderada pela população total em cada município. Posteriormente, foi realizada a análise multivariável por meio da regressão linear múltipla pelo método stepwise com significância 95\%.

Antes da realização da análise multivariável, foi feito o teste de correlação de Pearson com as variáveis independentes e optou-se pela exclusão de variáveis que tivessem colinearidade perfeita $(r>0,9)$, obtendo-se como resultado a exclusão de uma variável (renda per capita). Com isso, do total de 14 variáveis independentes, 13 continuaram no modelo para análise.

\section{Resultados}

A partir da análise descritiva, verificou-se que o Brasil teve em média $28,10 \%$ dos recursos do PNAE utilizados em compras de alimentos provenientes da agricultura familiar para alimentação escolar no período analisado e $11,98 \%$ de déficit de estatura em crianças menores de cinco anos atendidas nos serviços de atenção básica no mesmo período (Tabela 1). Além disso, observou-se que, em 2016, ocorreram, por um lado, redução em todas as regiões brasileiras no percentual de recursos utilizados para as compras da agricultura familiar para o PNAE e, por outro, aumento na prevalência de déficit de estatura em menores de cinco anos em todas as regiões brasileiras. 
TABELA 1

Percentual de compras da agricultura familiar para o PNAE e prevalência de déficit de estatura em menores de cinco anos

Brasil e regiões - 2013-2017

\begin{tabular}{|c|c|c|c|c|c|c|c|c|c|c|}
\hline \multirow[b]{2}{*}{ Regiões } & \multicolumn{10}{|c|}{ Percentual de compras da agricultura familiar para o PNAE } \\
\hline & $\begin{array}{c}\text { N. de } \\
\text { municípios }\end{array}$ & $\begin{array}{c}2013 \\
(\%)\end{array}$ & $\begin{array}{l}2014 \\
(\%)\end{array}$ & $\begin{array}{c}2015 \\
(\%)\end{array}$ & $\begin{array}{c}2016 \\
(\%)\end{array}$ & $\begin{array}{l}2017 \\
(\%)\end{array}$ & $\begin{array}{l}\text { Média } \\
(\%)\end{array}$ & DP & $p^{*}$ & Missing** \\
\hline Centro-Oeste & 413 & 18,4 & 18,2 & 19,3 & 18,2 & 19,9 & 18,8 & 16,8 & 0,001 & 11,6 \\
\hline Norte & 396 & 17,7 & 19,4 & 19,8 & 18,8 & 22,4 & 19,6 & 15,1 & 0,001 & 10,4 \\
\hline Nordeste & 1.659 & 17,9 & 20,1 & 21,7 & 22,1 & 22,6 & 20,9 & 14,8 & 0,001 & 7,5 \\
\hline Sudeste & 1.578 & 22,3 & 26,0 & 29,2 & 25,2 & 28,6 & 26,2 & 25,9 & 0,001 & 5,4 \\
\hline Sul & 1.187 & 34,8 & 36,6 & 42,1 & 41,1 & 47,5 & 40,4 & 20,8 & 1 & 0,3 \\
\hline \multirow[t]{2}{*}{ Brasil } & 5.107 & 22,8 & 25,6 & 27,9 & 26,5 & 29,4 & 28,1 & 17,2 & NA & 5,9 \\
\hline & \multicolumn{10}{|c|}{ Prevalência de déficit de estatura em menores de cinco anos } \\
\hline Regiões & $\begin{array}{c}\text { N. de } \\
\text { municípios }\end{array}$ & $\begin{array}{c}2013 \\
(\%)\end{array}$ & $\begin{array}{c}2014 \\
(\%)\end{array}$ & $\begin{array}{c}2015 \\
(\%)\end{array}$ & $\begin{array}{c}2016 \\
(\%)\end{array}$ & $\begin{array}{c}2017 \\
(\%)\end{array}$ & $\begin{array}{l}\text { Média } \\
(\%)^{\star \star \star}\end{array}$ & DP & ${ }^{\star} p$ & Missing ${ }^{\star \star}$ \\
\hline Centro-Oeste & 459 & 10,8 & 12,2 & 11,2 & 11,5 & 11,3 & 11,3 & 3,4 & 0,001 & 1,8 \\
\hline Norte & 396 & 17,0 & 17,4 & 15,6 & 16,1 & 15,8 & 16,3 & 5,0 & 0,001 & 12,0 \\
\hline Nordeste & 1.766 & 13,4 & 13,5 & 12,7 & 13,2 & 13,1 & 12,8 & 3,8 & 0,001 & 1,6 \\
\hline Sudeste & 1.648 & 9,8 & 10,0 & 9,5 & 9,6 & 9,5 & 11,4 & 4,3 & 0,001 & 1,2 \\
\hline Sul & 1.147 & 9,9 & 10,3 & 10,2 & 9,6 & 9,2 & 9,7 & 3,3 & 1 & 3,6 \\
\hline Brasil & 5.416 & 11,6 & 11,9 & 11,3 & 11,4 & 11,2 & 11,9 & 4,4 & NA & 2,7 \\
\hline
\end{tabular}

Fonte: Ministério da Educação. Fundo Nacional de Desenvolvimento da Educação; Ministério da Saúde. Sistema de Vigilância Alimentar e Nutricional (Sisvan). Elaboração dos autores.

Nota: * realizado teste $t$ para amostras independentes, em que o grupo de comparação foi a região Sul; DP = desvio padrão; $\mathrm{NA}=$ não se aplica; ${ }^{* \star}$ calculado sobre o total de municípios brasileiros (5.570) estratificados por região; ${ }^{\star * \star}$ ponderada pela população total da região.

Os resultados apontam diferenças regionais significativas ( $p<0,001)$, sendo que as regiões Sul e Sudeste apresentaram os maiores percentuais de compras de alimentos da agricultura familiar para o PNAE e também os menores percentuais de déficit de estatura. Já o Norte e Nordeste tiveram as menores médias de recursos usados nas compras e maiores no déficit de estatura em crianças menores de cinco anos, com 16,25\% e 12,84\%, respectivamente (Tabela 1 ).

As diferenças entre as médias de déficit de estatura nos municípios foram significativas em relação ao cumprimento legal do uso de $30 \%$ ou mais dos recursos do PNAE para compras de alimentos da agricultura familiar no período analisado, com uma diferença de 1,47 ponto percentual (Tabela 2). Ao analisar a correlação entre o déficit de estatura em menores de cinco anos e as variáveis contextuais, verificou-se que todas tiveram correlações significativas (Tabela 2). 
TABELA 2

Comparação de médias dos municípios brasileiros conforme adequação do uso de recursos para a compra de alimentos da agricultura familiar para o PNAE e déficit de estatura em menores de cinco anos e correlação entre a variável dependente e as variáveis independentes contextuais nos municípios brasileiros

Brasil - 2013-2017

\begin{tabular}{|c|c|c|c|c|c|}
\hline Variáveis & $\begin{array}{c}\text { N. de } \\
\text { municípios }\end{array}$ & Média & DP & $r$ & $p$ \\
\hline \multicolumn{6}{|l|}{ Independente principal } \\
\hline \multicolumn{6}{|l|}{$\begin{array}{l}\text { \% de déficit de estatura em menores cinco anos, segundo \% } \\
\text { de recursos para compra direta de alimentos da agricultura } \\
\text { familiar para o PNAE }\end{array}$} \\
\hline$<30 \%$ & 3.270 & 12,4 & 4,4 & NA & $<0,001^{\star}$ \\
\hline$\geq 30 \%$ & 2.145 & 11,0 & 3,9 & NA & $<0,001^{\star}$ \\
\hline \multicolumn{6}{|l|}{ Independentes contextuais } \\
\hline Taxa de mortalidade infantil (por mil nascidos vivos) & 5.416 & 19,2 & 7,1 & 0,3 & $<0,001^{\star \star \star}$ \\
\hline Índice de Gini & 5.416 & 0,4 & 0,1 & 0,4 & $<0,001^{\star \star \star}$ \\
\hline $\begin{array}{l}\text { \% dos ocupados no setor agropecuário - } 18 \text { anos ou mais } \\
2010\end{array}$ & 5.416 & 35,4 & 18,1 & 0,1 & $<0,001^{\star \star \star}$ \\
\hline \% da população em domicílios com água encanada 2010 & 5.416 & 85,8 & 14,4 & $-0,2$ & $<0,001^{\star \star \star}$ \\
\hline \% da população em domicílios com coleta de lixo 2010 & 5.413 & 94,3 & 10,3 & $-0,4$ & $<0,001^{\star \star \star}$ \\
\hline \% de crianças de 0 a 5 anos fora da escola 2010 & 5.406 & 59,9 & 11,2 & 0,1 & $<0,001^{\star \star \star}$ \\
\hline IDHM 2010 & 5.416 & 0,6 & 0,1 & $-0,4$ & $<0,001^{\star \star \star}$ \\
\hline Taxa de analfabetismo - 15 anos ou mais 2010 (\%) & 5.406 & 16,1 & 9,8 & 0,3 & $<0,001^{\star \star \star}$ \\
\hline Expectativa de anos de estudo aos 18 anos de idade em 2010 & 5.299 & 9,4 & 1,1 & $-0,3$ & $<0,001^{\star \star \star}$ \\
\hline Renda per capita (em reais) & 5.416 & 492,1 & 236,6 & $-0,4$ & $<0,001^{\star \star \star}$ \\
\hline Proporção de crianças extremamente pobres (\%) & 5.299 & 16,1 & 14,9 & 0,4 & $<0,001^{\star \star \star}$ \\
\hline Despesas per capita com agricultura - municipal (em reais) & 5.349 & 78,7 & 111,5 & $-0,1$ & $<0,001^{\star \star \star}$ \\
\hline Taxa de desocupação - dez anos ou mais (\%) & 5.406 & 6,7 & 3,7 & 0,2 & $<0,001^{\star \star \star}$ \\
\hline
\end{tabular}

Fonte: Ministério da Educação. Fundo Nacional de Desenvolvimento da Educação; Ministério da Saúde. Sistema de Vigilância Alimentar e Nutricional (Sisvan); PNUD; IBGE; Ipea. Elaboração dos autores.

Nota: * teste $t$ para amostras independentes; ${ }^{\star * \star}$ correlação de Pearson; NA = não se aplica.

A análise multivariável constatou que as variáveis com efeito sobre o déficit de estatura em crianças menores de cinco anos no modelo analisado foram: compra de alimentos da agricultura familiar para o PNAE; IDHM; Índice de Gini; taxa de analfabetismo; e proporção de crianças extremamente pobres.

0 modelo de regressão linear apresentado na Tabela 3 tem um poder explicativo de $24 \%$, mostrando que a cada ponto percentual de aumento no uso de recursos com a compra de alimentos da agricultura familiar para o PNAE pelo município haverá, em média, uma diminuição 0,55 ponto percentual na prevalência do déficit de estatura em menores de cinco anos, independentemente das demais variáveis do modelo. 
TABELA 3

Modelo de regressão linear múltipla para a variável déficit de estatura em menores de cinco anos, por municípios

Brasil - 2013-2017

\begin{tabular}{|c|c|c|c|c|c|}
\hline Variáveis & $\beta$ & $\begin{array}{c}\beta \\
\text { padronizado }\end{array}$ & IC (\%) & $p^{\star}$ & VIF \\
\hline $\begin{array}{l}\text { Déficit de estatura em menores de cinco } \\
\text { anos (constante) }\end{array}$ & 20,34 & NA & 17,28 a 23,32 & $<0,001$ & NA \\
\hline $\begin{array}{l}\text { \% de compra direta de alimentos da } \\
\text { agricultura familiar para o PNAE (V1) }\end{array}$ & $-0,553$ & $-0,058$ & $-0,786$ a 0,320 & $<0,001$ & 1,06 \\
\hline IDHM (V2) & $-22,69$ & $-0,341$ & $-26,61$ a 18,78 & $<0,001$ & 6,25 \\
\hline $\begin{array}{l}\text { Taxa de analfabetismo - } 15 \text { anos ou mais } \\
2010 \text { (V3) }\end{array}$ & $-0,135$ & $-0,283$ & $-0,162$ a $-0,109$ & $<0,001$ & 5,57 \\
\hline Índice de Gini (V4) & 14,96 & 0,197 & 12,66 a 17,25 & $<0,001$ & 1,64 \\
\hline $\begin{array}{l}\text { Proporção de crianças extremamente pobres } \\
\text { (V5) }\end{array}$ & 0,078 & 0,250 & 0,06 a 0,096 & $<0,001$ & 6,22 \\
\hline \multicolumn{6}{|c|}{$\begin{array}{l}\text { Equação: } Y=20,34-0,553 \mathrm{~V} 1-22,69 \mathrm{~V} 2-0,135 \mathrm{~V} 3+14,46 \mathrm{~V} 4+0,078 \mathrm{~V} 5 \\
\text { Modelo }=F(5,5293)=328,2, p=\left\langle 0,001^{\star *}, R^{2} \text { ajustado }=0,237 \text { e Durbin- }\right. \\
\text { Watson }=1,718\end{array}$} \\
\hline
\end{tabular}

Fonte: Ministério da Educação. Fundo Nacional de Desenvolvimento da Educação; Ministério da Saúde. Sistema de Vigilância Alimentar e Nutricional (Sisvan); PNUD; IBGE; Ipea. Elaboração dos autores.

Nota: ${ }^{*}$ teste $t ;{ }^{* *}$ ANOVA.

\section{Discussão}

Os resultados do presente estudo reafirmam os efeitos dos indicadores socioeconômicos sobre a redução da prevalência do déficit de estatura em crianças menores de cinco anos atendidas nos serviços de atenção básica nos municípios brasileiros. Entretanto, é importante destacar a associação entre a compra de alimentos da agricultura familiar e o déficit de estatura em menores de cinco anos, pois, conforme apresentado na Tabela 1, a média do déficit de estatura em crianças menores de cinco anos atendidas na atenção básica foi menor nos municípios com utilização de $30 \%$ ou mais dos recursos do PNAE para a compra de alimentos da agricultura familiar. Ademais, na análise multivariável, verificou-se que a compra de alimentos da agricultura familiar para o PNAE pode contribuir para a redução da prevalência do déficit de estatura em crianças menores de cinco anos mesmo com a presença de variáveis contextuais, apesar de essas últimas terem maior efeito nas prevalências de déficit de estatura no modelo apresentado (Tabela 3).

Esse resultado corrobora aquele apresentado em um ensaio comunitário realizado em Gana, por Gelli et al. (2016), com o objetivo de analisar os impactos de modelos alternativos para alimentação escolar sobre a saúde de crianças e adolescentes, mostrando em seus resultados diferenças significativas nos grupos controle (sem alimentação escolar) e teste (com alimentação escolar e incentivo à agricultura familiar), sendo que as crianças e adolescentes que faziam parte do grupo teste tiveram melhor estado nutricional avaliado a partir dos índices altura para idade e IMC para idade. Além disso, nas comunidades do grupo teste, ocorreu um aumento na produção e venda de alimentos produzidos localmente ao longo do período do estudo (GELLI et al., 2016). 
Nesse contexto no Brasil, o PNAE é um programa público que vem contribuindo para o fortalecimento da agricultura familiar, pois gerou um círculo propulsor do desenvolvimento local, como também melhorou a qualidade da alimentação oferecida aos estudantes, o que contribui para a SAN em seus diferentes aspectos, desde o acesso aos alimentos in natura provenientes da produção local até uma transformação no aspecto da promoção à alimentação saudável e adequada nas escolas por meio da inserção de ações de educação alimentar e nutricional e do tema alimentação saudável nos projetos pedagógicos das escolas, como preconizado na legislação que dá suporte ao programa (OPAS, 2017; BRASIL, 2013a).

Estudo realizado por Gonçalves et al. (2015), para municípios do Rio de Janeiro e São Paulo, demonstrou melhor qualidade nutricional dos cardápios nas escolas após a aquisição de alimentos da agricultura familiar pelos municípios, com redução do percentual de cardápios com classificação ruim e aumento da classificação boa ou ótima, conforme o Índice de Qualidade dos Cardápios da Alimentação Escolar. Alvarez (2017) também verificou, para o Estado de São Paulo, que os municípios com aquisição de alimentos da agricultura familiar tiveram melhor qualidade nutricional dos cardápios, com maior oferta de alimentos básicos (in natura e minimamente processados) e menor utilização de alimentos restritivos (doces).

Em consequência do perfil supracitado, o PNAE tem sido referência para outros países por sua abordagem intersetorial, pelo acesso dos estudantes a alimentos saudáveis de maneira gratuita, bem como pela promoção de mercados locais de compra de alimentos, priorizando os pequenos produtores rurais, contribuindo, assim, para a estruturação de sistemas alimentares mais sustentáveis e saudáveis, pois incentiva a compra de alimentos in natura e de base agroecológica (SANTARELLI et al., 2019).

Nos resultados da análise descrita na presente pesquisa constatou-se, ainda, que a média do percentual de recursos do $\operatorname{PNAE}(28,10 \%)$ utilizados com compras de alimentos da agricultura familiar está abaixo do que rege a legislação no Brasil e com diferenças regionais significativas, em que Sul e Sudeste se destacaram com maiores percentuais de compra.

Esses resultados corroboram o estudo de Machado et al. (2018) que avaliou a compra de alimentos da agricultura familiar para o PNAE em 2011 nos municípios brasileiros, relatando que a região Sul apresentava o maior percentual de municípios realizando a compra, enquanto o menor valor foi encontrado no Centro-Oeste. Além disso, o mesmo estudo verificou que os municípios com menor frequência de compra eram aqueles com grande porte populacional (mais de 100.000 habitantes), gestão dos recursos do programa do tipo mista, descentralizada ou terceirizada e que não tinham nutricionistas como responsáveis técnicos na alimentação escolar (MACHADO et al., 2018).

Ainda a partir da análise descritiva também observou-se que, no período analisado, o Brasil apresentou uma média de 11,98\% de déficit de estatura em crianças menores de cinco anos, a qual, de acordo com o indicador da Ripsa (2012) no Brasil, está acima da meta de déficit de estatura em crianças nessa faixa de idade, que deve ser inferior a $10 \%$. 
Além disso, foi constatada uma diferença regional nas médias de déficit de estatura, com as maiores prevalências no Norte $(16,25 \%)$ e Nordeste $(12,84 \%)$, enquanto o Sul $(9,71 \%)$ foi a única região a atingir a meta nacional, com média de $10 \%$. Logo, é importante ressaltar que o déficit de estatura reflete a desnutrição crônica, sendo que em todos os resultados apresentados no país os percentuais estão acima do esperado para uma população saudável de referência, conforme a proposta da curva de crescimento da WHO (2006), que coloca um ponto de corte abaixo de $2,3 \%$ em populações saudáveis (PEREIRA et al., 2017; BRASIL, 2011). Isso mostra que o déficit de estatura em crianças menores de cinco anos ainda é um problema de saúde pública no país.

Nesse cenário, os inquéritos populacionais realizados anteriormente no Brasil mostravam uma redução no déficit de estatura em crianças entre 5 e 9 anos (IBGE, 2010). Estudo realizado por Pereira et al. (2017) utilizando dados da Pesquisa de Orçamentos Familiares (POF) 2008-2009 demostrou que, entre 1975 e 2009, a prevalência de déficit de estatura reduziu-se de $29,3 \%$ para $7,2 \%$, em meninos, e de $26,7 \%$ para $6,3 \%$, em meninas. Esses declínios ocorreram de maneira diferente entre as regiões brasileiras, com maior decréscimo no Sul, Sudeste e Centro-Oeste, enquanto o Norte e o Nordeste tiveram as maiores prevalências do déficit de estatura, embora o Nordeste tenha resultados próximos ao eixo Centro-Sul do país, o que corrobora os resultados do presente estudo (PEREIRA et al., 2017).

Pereira et al. (2017) também mostraram que a maior chance de déficit de estatura ocorreu em crianças indígenas, sendo quase o dobro de chances. 0 mesmo estudo constatou que, quanto menor o nível de renda, maior é a chance de a criança apresentar déficit de estatura, sendo quase dez vezes superior para o grupo de até $1 / 4$ de salário mínimo (PEREIRA et al., 2017).

Outro estudo realizado em um município do Acre, na região Norte (MANTOVANI et al., 2016), obteve uma prevalência de $14,4 \%$ de déficit de estatura em menores de cinco anos em 2011. Entre os fatores que mais contribuíram para o déficit de estatura nas crianças avaliadas, também estavam as condições socioeconômicas, as características maternas e a falta de instalações sanitárias adequadas. Em relação a este último fator, é conhecida a sua relação com piores condições socioeconômicas, sendo que a falta de saneamento básico torna a criança mais propensa a doenças diarreicas que, por sua vez, podem gerar a redução do crescimento linear (MANTOVANI et al., 2016; SILVA-JOVENTINO, 2013).

Como relatado anteriormente, a desnutrição traz consequências negativas para a saúde da criança e aumenta a taxa de mortalidade infantil, pois pode causar danos físicos e/ou cognitivos, entre estes o menor desempenho escolar e a maior propensão a doenças. Assim, as crianças desnutridas e com menor rendimento escolar poderão na vida adulta ter menor capacidade para o trabalho e, consequentemente, menor renda. Portanto, a desnutrição na infância contribui para a transmissão intergeracional da pobreza (RAMOS; LIMA, 2015; RAMOS; DUMITH; CESAR, 2015).

Sabe-se que programas voltados para acabar com a fome e promover a SAN podem contribuir para a redução da desnutrição infantil (MARTINS, 2013; PEREIRA et al., 2017). 
Em um estudo realizado por Monteiro et al. (2009), foi constatada uma redução de $30 \%$ da desnutrição em crianças menores de cinco anos cujas famílias recebiam o benefício do programa. Esse impacto ocorreu em virtude de as famílias de baixa renda estarem utilizando o recurso para gasto domiciliar com alimentação, consequentemente aumentando a disponibilidade e o acesso aos alimentos (PEREIRA et al., 2017; MARTINS, 2013).

Isso demostra a necessidade da continuidade de políticas públicas voltadas para a redução da desnutrição, corroborando a Declaração de Roma sobre Nutrição, em 2014, que coloca o combate à desnutrição infantil como prioridade na agenda política internacional (WHO, 2014a).

Entre os tratados internacionais que têm o objetivo de reduzir a desnutrição, citamos - "Plano de implementação sobre nutrição materna, lactantes e crianças pequenas", proposto e aprovado pela OMS e com vigência de 2012 a 2025. Uma de suas metas é a redução em $40 \%$ do número de crianças com atraso no crescimento no mundo, utilizando como parâmetro base o percentual de 2010 (WHO, 2014b).

Ainda neste plano são propostas cinco linhas de ação: criar um ambiente propício à implementação de políticas abrangentes de alimentação e nutrição; incluir nos planos nacionais de nutrição intervenções sanitárias eficazes que tenham efeitos na nutrição; estimular o desenvolvimento de políticas e programas fora do setor da saúde que reconheçam e incluam a nutrição; fornecer recursos humanos e financeiros suficientes para a implementação de intervenções nutricionais; e monitorar e avaliar a implementação das políticas e programas (WHO, 2014b).

Entre os pontos-chave para o alcance dessas proposições está a modificação dos sistemas alimentares globais para um modelo que priorize a proteção à saúde humana e ao meio ambiente. Por outro lado, o modo atual de organização da cadeia produtiva de alimentos tem gerado impactos negativos na saúde, pois as práticas agrícolas voltadas para abastecer a indústria de alimentos com base em uma agricultura com uso intensivo de produtos químicos, na produção intensiva de gado, na produção e comercialização em massa de alimentos ultraprocessados e no desenvolvimento de cadeias de alimentos baseadas em monoculturas e com o mercado concentrado em poucas multinacionais não favorecem a soberania alimentar dos povos e, consequentemente, o uso sustentável do meio ambiente (IPES-FOOD, 2017).

No cenário mundial vivem-se os impactos das mudanças climáticas que tendem a ser cada vez mais graves em virtude da sindemia global - termo utilizado para se referir à sinergia existente no espaço e no tempo das três pandemias - obesidade, desnutrição e mudanças climáticas - que vêm afetando significantemente a população, sendo as duas primeiras, em conjunto com outras causas nutricionais, responsáveis por cerca de $19 \%$ do adoecimento e mortes prematuras no mundo (IDEC, 2019).

Em seu primeiro relatório temático, From uniformity to diversity: a paradigm shift from industrial agriculture to diversified agroecological systems, a IPES-Food (2016) identificou uma mudança de paradigma rumo a sistemas agroecológicos diversificados e apontou 
como ponto-chave para enfrentar os impactos negativos de nossos sistemas alimentares globais no meio ambiente e na sociedade os sistemas agroecológicos diversificados, um modelo baseado na biodiversidade, preservação ambiental e justiça social.

A promoção de modelos agrícolas alternativos, como a agricultura familiar, contribui para o que é denominada de "agricultura sensível à nutrição", sendo este termo orientado na perspectiva dos determinantes sociais da saúde que consideram a agricultura de base agroecológica uma prioridade para o alcance da SAN (MALUF et al., 2015; RIBEIRO; JAIME; VENTURA, 2017). A agricultura sensível à nutrição, ao promover os circuitos alimentares curtos, valorizar a cultura alimentar e a biodiversidade, gerando a autonomia dos agricultores em relação às grandes indústrias, contribui para a diversidade de alimentos produzidos e consumidos, unindo as pontas entre produção e consumo de uma dieta saudável (RIBEIRO; JAIME, VENTURA,2017).

Todas as recomendações e metas citadas reforçam a importância de programas como o PNAE, que estimula a agricultura familiar por meio das compras institucionais, promovendo sistemas alimentares mais sustentáveis e saudáveis. Logo, diante dos resultados apresentados neste estudo, se fazem necessárias ações dos gestores do PNAE no Brasil no sentido de melhorar as estratégias de compras de alimentos da agricultura familiar, tendo em vista os seus efeitos positivos sobre a saúde dos escolares, contribuindo para melhorar o estado nutricional das crianças e dos agricultores familiares por meio da promoção da SAN e do DHAA.

Apesar de as evidências históricas comprovarem que o investimento em agricultura familiar, aliado a políticas públicas, contribui para a SAN, no atual cenário das políticas públicas brasileiras vem ocorrendo queda de investimentos na agricultura familiar, que coincidiu com a redução do percentual de recursos utilizados para a compra de alimentos da agricultura familiar, entre 2015 e 2016, no PNAE observadas no presente estudo. Esse cenário pode ser consequência do decréscimo nos investimentos públicos neste setor, pois os recursos executados para as ações de promoção e fortalecimento da agricultura familiar no Brasil diminuíram de $\mathrm{R} \$ 13,05$ milhões, em 2014, para $\mathrm{R} \$ 3,36$ milhões, em 2017 (BRASIL, 2018), com impactos negativos para a SAN dada a magnitude do corte orçamentário. Logo, são importantes a ampliação e o incentivo às políticas de fortalecimento da agricultura familiar, a fim de alcançar impactos positivos para o desenvolvimento econômico e ambiental sustentáveis e colaborar para reduzir as desigualdades sociais tão proeminentes no Brasil.

Diante dos resultados expostos nessa pesquisa, é relevante que sejam realizados estudos locais com avaliação dos fatores que contribuem para o déficit de estatura nas crianças menores de cinco anos, a fim de propor estratégias para mudanças deste indicador nos municípios. Como constatado no presente estudo, os fatores socioeconômicos têm maior impacto sobre este problema e, portanto, políticas e programas que visem diminuir as desigualdades sociais e melhorar as condições de vida da população são extremamente necessários para a redução da desnutrição infantil. 
Uma possibilidade que já existiu no Brasil e poderia ser retomada para monitoramento do desnutrição infantil em menores de cinco anos é a Agenda para Intensificação da Atenção Nutricional à Desnutrição Infantil (Andi), que tinha como foco os municípios brasileiros com menos de 150 mil habitantes com prevalência igual ou superior a $10 \%$ de desnutrição em crianças menores de cinco anos, identificada pelo índice baixo peso para a idade, conforme os dados do Sistema de Vigilância Alimentar e Nutricional (Sisvan) de 2011. Essa estratégia baseava-se em ações de estruturação e qualificação das ações de atenção à saúde da criança, especialmente no âmbito da atenção básica, e ocorreu de 2012 a 2015 (BRASIL, 2013b).

O presente trabalho possui um delineamento ecológico com uso de dados secundários que são uma boa opção metodológica para quem se propõe a analisar o impacto de um programa público sobre indicadores de saúde (déficit de estatura). Mas devemos relatar algumas limitações do estudo, como a cobertura dos dados do Sisvan no período de 2013 a 2017 para a faixa etária analisada, pois foram coletados os dados antropométricos de cerca de 4.488.230 crianças menores de cinco anos atendidas na atenção básica nesse período, o que corresponde a 30,4\% de cobertura (SISVAN, 2020; DATASUS, 2020).

Além disso, os dados coletados no Sisvan, no período analisado, eram em sua maioria $(72 \%)$ de crianças beneficiárias do Programa Bolsa Família, ou seja, os resultados apresentados têm maior foco em crianças em situação de vulnerabilidade social, que são as frequentadoras de estabelecimentos escolares públicos; logo, deve-se ter cautela ao extrapolar esses resultados para toda a população brasileira.

Apesar disso, pode-se relatar a validade externa desta pesquisa para os diferentes municípios brasileiros e países que possuem programas com estratégias semelhantes às utilizadas pelo PNAE, em relação às compras institucionais da agricultura familiar para alimentação escolar.

\section{Conclusão}

Os resultados da presente pesquisa mostraram que a utilização dos recursos do PNAE para a compra de alimentos da agricultura familiar ainda está abaixo dos $30 \%$ preconizados pela legislação e o déficit de estatura está com prevalência alta entre as crianças menores de cinco anos atendidas nos serviços de atenção básica no Brasil. Além disso, foi verificado que a compra de alimentos da agricultura familiar para alimentação escolar no Brasil pode contribuir para a redução na prevalência do déficit de estatura em crianças menores de cinco anos, mesmo que em menor grau se comparada aos fatores contextuais analisados.

\section{Referências}

AKOMBI, B. J. et al. Stunting and severe stunting among children under-5 years in Nigeria: a multilevel analysis. BMC Pediatrics, v. 17, n. 1, 2017. Disponivel em: https://doi.org/10.1186/ s12887-016-0770-z. Acesso em: 07 jul. 2020. 
ALVAREZ, D. B. Efeito da Lei Federal 11.947/09 na qualidade dos cardápios propostos pelo Programa de Alimentação Escolar do estado de São Paulo. Dissertação (Mestrado em Nutrição e Saúde Pública) - Faculdade de Saúde Pública, USP, São Paulo, 2017.

BRASIL. Presidência da República. Lei n. 11.947 de 16 de junho de 2009. Dispõe sobre o atendimento da alimentação escolar e do Programa Dinheiro Direto na Escola aos alunos da educação básica. Diário Oficial da União, 16 de junho 2009.

BRASIL. Ministério da Saúde. Orientações para coleta e análise de dados antropométricos em serviços de saúde. Brasília, 2011. Disponível em: http://189.28.128.100/dab/docs/portaldab/ publicacoes /orientacoes_coleta_analise_dados_antropometricos.pdf. Acesso em: 20 abr. 2020.

BRASIL. Ministério da Educação. Resolução n. 26 de 17 de junho de 2013. Dispõe sobre o atendimento da alimentação escolar aos alunos da educação básica no âmbito do Programa Nacional de Alimentação Escolar (PNAE). Diário Oficial da União, 17 de junho 2013a.

BRASIL. Ministério da Saúde. Manual instrutivo para implementação da Agenda para Intensificação da Atenção Nutricional à Desnutrição Infantil: Portaria n. 2.387, de 18 de outubro de 2012. Brasília, 2013b. Disponível em: http://189.28.128.100/dab/docs/ portaldab / publicacoes/ manual_andi.pdf. Acesso em: 07 dez. 2020.

BRASIL. Ministério da Saúde. Sistema de Vigilância Alimentar e Nutricional: relatórios 2020. Disponível em: https://sisaps.saude.gov.br/sisvan/relatoriopublico/index. Acesso em 3 maio 2020.

BRASIL. Portal da transparência, 2018. Disponível em: www3.transparencia.gov.br/programase-acoes/acao/210V-promocao-e-fortalecimento-da-agricultura-familiar?ano=2017. Acesso em: 28 ago. 2020.

DATASUS. Informações de saúde. 2020. Disponível em: http://www2.datasus.gov.br/DATASUS/ index.php?area=0206. Acesso em: 07 out. 2020.

FAO - Food and Agriculture Organization of the United Nations et al. El estado de la seguridad alimentaria y la nutrición en el mundo. Roma: FAO, 2019. Disponível em: https://docs.wfp.org/ api/documents/WFP-0000106773/download/?_ga=2.108888833.2060569887.15841141002139328721.1584114100. Acesso em: 20 dez. 2020.

FAO - Food and Agriculture Organization of the United Nations. The state of food insecurity in the world: the multiple dimensions of food security. Roma: FAO, 2013. Disponivel em: http:// www.fao.org/3/a-i3434e.pdf. Acesso em: 20 dez. 2020.

FAO - Food and Agriculture Organization of the United Nations. 0 estado da segurança alimentar e nutricional no Brasil: um retrato multidimensional. Brasília: FAO, 2014. Disponível em: http:// www.fao.org.br/ download/SOFI_p.pdf. Acesso em 26 jun. 2020.

FNDE - Fundo Nacional de Desenvolvimento da Educação. Consultas de dados físicos e financeiros do PNAE. Disponivel em: https://www.fnde.gov.br/index.php/programas/pnae/ pnae-consultas/pnae-dados-fisicos-e-financeiros-do-pnae. Acesso em 5 dez. 2020.

FNDE - Fundo Nacional de Desenvolvimento da Educação. PNAE - Programa Nacional de Alimentação Escolar. Dados da Agricultura Familiar, 2019. Disponível em: https://www.fnde. gov.br/programas/pnae/pnae-consultas/pnae-dados-da-agricultura-familiar. Acesso em: 09 dez. 2020.

GARCIA, L. R. S. Déficit estatural infantil em beneficiários do Programa Bolsa Família: análise dos determinantes sociais e da evolução da desigualdade no Brasil. Tese (Doutorado em Saúde Coletiva) - Programa de Pós-Graduação em Saúde Coletiva da UFRN, Natal, 2018. 
GELLI, A. et al. Evaluation of alternative school feeding models on nutrition, education, agriculture and other social outcomes in Ghana: rationale, randomised design and baseline data. Trials, v. 17, 2016. Disponível em: https://link.springer.com/article/ 10.1186/s13063-015-1116-0. Acesso em: 12 jun. 2020.

GONÇALVES, H. V. B. et al. Family farming products on menus in school feeding: a partnership for promoting healthy eating. Ciência Rural, v.45, n. 12, p. 2267-2273, 2015.

IDEC - Instituto Brasileiro de Defesa do Consumidor. A sindemia global da obesidade, desnutrição e mudanças climáticas: um sumário executivo da comissão The Lancet, 2019. Disponível em: https://crn5.org.br/wp-content/uploads/Vers\%c3\%a3o-portugu\%c3\%aas-LANCET-2019Sindemia_compressed-1.pdf. Acesso em: 7 dez. 2020.

IBGE - Instituto Brasileiro de Geografia e Estatística. Pesquisa de Orçamentos Familiares 20082009: antropometria e estado nutricional de crianças, adolescentes e adultos no Brasil. Rio de Janeiro, 2010. Disponível em: https://biblioteca.ibge.gov.br/visualizacao/livros/liv45419.pdf. Aceso em 20 nov. 2019.

IPES-FOOD - International Panel of Experts on Sustainable Food Systems. From uniformity to diversity: a paradigm shift from industrial agriculture to diversified agroecological systems. 2016. Disponível em: www.ipes-food.org. Acesso em: 7 dez. 2020.

IPES-FOOD - International Panel of Experts on Sustainable Food Systems. Unravelling the food-health nexus: addressing practices, political economy, and power relations to build healthier food system. 2017. Disponivel em: http://www.ipes-food.org/_img/\%20upload/files/ Health_FullReport(1).pdf. Acesso em: 7 abr. 2020.

MACHADO, P. M. de O. et al. Compra de alimentos da agricultura familiar pelo Programa Nacional de Alimentação Escolar (PNAE): estudo transversal com o universo de municípios brasileiros. Ciência \& Saúde Coletiva, Rio de Janeiro, v. 23, n. 12, p. 4153-4164, dez. 2018. Disponível em: http://www.scielo.br/scielo.php?script=sci_arttext\&pid=S1413-81232018001204153\&lng=en\& nrm=iso. Acesso em: 20 maio 2021.

MALUF, R. S. et al. Nutrition-sensitive agriculture and the promotion of food and nutrition sovereignty and security in Brazil. Ciência \& Saúde Coletiva, Rio de Janeiro, v. 20, n. 8, p. 23032312, ago. 2015. Disponível em: http://www.scielo.br/scielo.php?script=sci_arttext\&pid=S1413$81232015000802303 \&$ lng=en\&nrm=iso. Acesso em: 20 maio 2021.

MANTOVANI, S. A. S. et al. Stunting in children under five years old is still a health problem in the Western Brazilian Amazon: a population-based study in Assis Brasil, Acre, Brazil. Ciência \& Saúde Coletiva, Rio de Janeiro, v. 21, n. 7, p. 2257-2266, jul. 2016. Disponível em: http://www. scielo.br/scielo.php? script=sci_arttext\&pid=S1413-81232016000702257 \&lng=en\&nrm=iso. Acesso em: 20 maio 2021.

MARTINS A. P. B. Impacto do Programa Bolsa Família sobre a aquisição de alimentos em famílias brasileiras de baixa renda. Tese (Doutorado em Ciências) - Universidade de São Paulo (USP), São Paulo, 2013.

MONTEIRO, C. A. et al. Causas do declínio da desnutrição infantil no Brasil, 1996-2007. Revista de Saúde Pública, São Paulo, v. 43, n. 1, p. 35-43, fev. 2009. Disponível em: http://www.scielo. $\mathrm{br} / \mathrm{scielo}$.php? script=sci_arttext\&pid=S0034-89102009000100005\&lng=en\&nrm=iso. Acesso em: 20 maio 2021.

ONIS, M.; BLÖSSNER, M.; BORGHI, E. Prevalence and trends of stunting among pre-school children, 1990-2020. Public Health Nutrition, v. 15, n. 1, 2011. 
OPAS - Organização Pan-Americana da Saúde. Sistemas alimentares e nutrição: a experiência brasileira para enfrentar todas as formas de má nutrição. Brasília, DF: Opas, 2017. Disponível em: http://iris.paho.org/xmlui/handle/123456789/34289. Acesso em: 12 nov. 2019.

PEDRAZA, D. F.; SALES, M. C.; MENEZES, T. N. de. Fatores associados ao crescimento linear de crianças socialmente vulneráveis do Estado da Paraíba, Brasil. Ciência \& Saúde Coletiva, Rio de Janeiro, v. 21, n. 3, p. 935-946, mar. 2016. Disponivel em: http://www.scielo.br/scielo. php?script=sci_arttext\&pid=S1413-81232016000300935\&lng=en\&nrm=iso. Acesso em: 20 fev. 2021.

PEIXINHO, A. M. L. A trajetória do Programa Nacional de Alimentação Escolar no período de 2003-2010: relato do gestor nacional. Ciência \& Saúde Coletiva, Rio de Janeiro, v. 18, n. 4, p. 909-916, 2013.

PEREIRA, I. F. da S. et al. Estado nutricional de menores de 5 anos de idade no Brasil: evidências da polarização epidemiológica nutricional. Ciência \& Saúde Coletiva, Rio de Janeiro, v. 22, n. 10, p. 3341-3352, out. 2017. Disponível em: http://www.scielo.br/scielo.php?script=sci_ arttext\&pid=S1413-81232017021003341 \&lng=en\&nrm=iso. Acesso em: 20 maio 2021.

RAMOS, C. V.; DUMITH, S. C.; CESAR, J. A. Prevalência e fatores associados ao déficit de altura e excesso de peso em crianças de 0 a 5 anos do semiárido. Jornal de Pediatria, Rio de Janeiro, v. 91, n. 2, p. 175-182, abr. 2015. Disponível em: http://www.scielo.br/scielo.php?script=sci_ arttext\&pid=S0021-75572015000200175\&lng=en\&nrm=iso. Acesso em: 20 maio 2021.

RAMOS, M. K. P.; LIMA, A. M. C. de; GUBERT, M. B. Agenda para intensificação da atenção nutricional à desnutrição infantil: resultados de uma pactuação interfederativa no Sistema Único de Saúde. Revista de Nutrição, Campinas, v. 28, n. 6, p. 641-653, 2015. Disponível em: http:// www.scielo.br/scielo.php?script=sci_arttext\&pid=S1415-52732015000600641\&lng=en\&nrm= iso. Acesso em: 22 maio 2020.

RIBEIRO, H.; JAIME, P. C.; VENTURA, D. Alimentação e sustentabilidade. Estudos Avançados, São Paulo, v. 31, n. 89, p. 185-198, abr. 2017. Disponivel em: http://www.scielo.br/scielo. php?script=sci_arttext\&pid=S0103-40142017000100185\&lng=en\&nrm=iso. Acesso em: 20 maio 2021. https://doi.org/10.1590/s0103-40142017.31890016.

RIPSA - Rede Interagencial de Informações para a Saúde no Brasil. Ficha de qualificação: prevalência de déficit estatural para a idade em crianças menores de 5 anos de idade. 2012. Disponível em: http://fichas.ripsa.org. br/2012/g-11/?l=pt_BR. Acesso em: 10 abr. 2020.

SANTARELLI, M. et al. Informe Dhana 2019: autoritarismo, negação de direitos e fome. Brasília: Fian, 2019. Disponível em: https://fianbrasil.org.br/wp-content/uploads/2019/11/InformeDhana-2019_v-final.pdf. Acesso em: 20 fev. 2020.

SILVA-JOVENTINO, E. et al. Condiciones sociodemográficas y de salud para auto-eficacia materna en la prevención de la diarrea infantil. Revista de Salud Pública, v. 15, n. 4, 2013. Disponível em: https://www.scielosp.org/pdf/rsap/2013.v15n4/592-604. Acesso em: 20 mar. 2020.

SISVAN - Sistema de Vigilância Alimentar e Nutricional. Relatórios estado nutricional, 2020. Disponivel em: http://sisaps.saude.gov.br/sisvan/. Acesso em: 7 dez. 2020.

SBP - Sociedade Brasileira de Pediatria. Avaliação nutricional da criança e do adolescente. São Paulo: Departamento de Nutrologia, 2009.

WHO - World Health Organization. Segunda Conferencia Internacional sobre Nutrición. Documento final de la Conferencia: Declaración de Roma sobre la Nutrición. Roma, $2014 a$. Disponivel em: http://www.fao.org/3/a-ml542s.pdf. Acesso em: 7 dez. 2020. 
WHO - World Health Organization. Plan de aplicación integral sobre nutrición materna, del lactante y del niño. Ginebra, 2014b. Disponível em: https://www.who.int/nutrition/ publications/ CIP_document/es/. Acesso em: 7 dez. 2020.

WHO - World Health Organization. WHO child growth standards: length/height-for-age, weight-for-age, weight-for-length, weight-forheight and body mass index-for-age: methods and development. Geneva, 2006. Disponivel em: https://www.who.int/childgrowth/standards/ technical_report/en/. Acesso em: 20 dez. 2020.

\title{
Sobre os autores
}

Genykléa Silva de Oliveira é nutricionista, doutora em Saúde Coletiva pelo Programa de Pós-Graduação em Saúde Coletiva da Universidade Federal do Rio Grande do Norte (PPGSCOL/ UFRN). Nutricionista da Secretaria de Estado da Saúde Pública do Rio Grande do Norte (Sesap$\mathrm{RN}$ ) e professora da Unifacex.

Clelia de Oliveira Lyra é nutricionista. Professora doutora do Departamento de Nutrição da Universidade Federal do Rio Grande do Norte (UFRN).

Angelo Giuseppe Roncalli da Costa Oliveira é graduado em odontologia pela Universidade Federal do Rio Grande do Norte (UFRN). Professor doutor do Departamento de Odontologia da UFRN.

Maria Angela Fernandes Ferreira é cirurgiã-dentista. Professora doutora do Departamento de Odontologia da Universidade Federal do Rio Grande do Norte (UFRN).

\section{Endereço para correspondência}

\author{
Genykléa Silva de Oliveira \\ UniFacex - Centro Universitário Facex \\ Rua Orlando Silva, 2897, Capim Macio \\ 59080-020 - Natal-RN, Brasil \\ Clelia de Oliveira Lyra \\ Departamento de Nutrição da UFRN \\ Av. Senador Salgado Filho, s/n, Lagoa Nova \\ 59078-970 - Natal-RN, Brasil \\ Angelo Giuseppe Roncalli da Costa Oliveira \\ Departamento de Odontologia da UFRN \\ Av. Senador Salgado Filho, 1787, Lagoa Nova \\ 59056-000 - Natal-RN, Brasil \\ Maria Angela Fernandes Ferreira \\ Departamento de Odontologia da UFRN \\ Av. Senador Salgado Filho, 1787, Lagoa Nova \\ 59056-000 - Natal-RN, Brasil
}

\section{Abstract}

Reduction of stature deficit and purchase of family agriculture produce for school meals in Brazil

The objective of this work is to analyze the effect of direct purchase of food from family farms for school meals on the deficit of stature in children under 5 years of age between 2013 and 2017 in 
Brazil. Method: Ecological and longitudinal study in which data collection occurred in different databases. The analysis was performed using the t-test for independent samples in order to compare the means between the variables stature deficit and purchases from family agriculture. In addition, a multivariable analysis was conducted through multiple linear regression. Results: There was a difference in means in the prevalence of significant stature deficit between the municipalities that bought $<30 \%$ and $\geq 30 \%$ of food from family farms for school feeding, with 1.47 points of difference. The linear regression model showed that for each percentage point of increase in the purchase of food from family farms for school feeding by the municipality, there will be on average a decrease of 0.55 points in the prevalence of stature deficit, independent of the other variables. Conclusion: the purchase of food from family agriculture for school feeding in Brazil may contribute to the reduction of the prevalence of stature deficit in children under five years old.

Keywords: Food and nutritional security. Child malnutrition. School feeding. Agriculture.

\section{Resumen}

Reducción del déficit de estatura y de la agricultura familiar en la alimentación escolar en Brasil

Objetivo: Analizar el efecto de la compra directa de alimentos de las granjas familiares para la alimentación escolar en el déficit de estatura de los niños menores de cinco años entre 2013 y 2017 en Brasil. Método: Estudio ecológico y longitudinal en el que se recogieron datos en diferentes bases de datos. El análisis se llevó a cabo mediante la prueba $t$ para muestras independientes con el fin de comparar las medias entre las variables déficit de estatura y compras de la agricultura familiar. Además, se hizo un análisis multivariable a través de una regresión lineal múltiple. Resultados: Se produjo una diferencia de medias en la prevalencia del déficit de estatura significativo entre los municipios que compraron $<30 \%$ y $\geq 30 \%$ de los alimentos de las granjas familiares para la alimentación escolar, con 1,47 puntos de diferencia. El modelo de regresión lineal mostró que por cada punto porcentual de aumento en la compra de alimentos de las granjas familiares para la alimentación escolar por parte del municipio, habrá, en promedio, una disminución de 0,55 puntos en la prevalencia del déficit de estatura, independientemente de las demás variables. Conclusión: la compra de alimentos de las granjas familiares para la alimentación escolar en el Brasil puede contribuir a reducir la prevalencia del déficit de estatura en los niños menores de cinco años.

Palabras clave: Seguridad alimentaria y nutricional. Desnutrición infantil. Alimentación escolar. Agricultura. 
Anexo

\section{QUADRO 1}

Descrição das variáveis de estudo

\begin{tabular}{|c|c|c|c|}
\hline Variável & Descrição & Tipo & $\begin{array}{c}\text { Fonte de } \\
\text { dados }\end{array}$ \\
\hline \multicolumn{4}{|l|}{$\begin{array}{l}\text { Variável desfecho - } \\
\text { estado nutricional }\end{array}$} \\
\hline $\begin{array}{l}\text { Percentual de crianças } \\
\text { menores de cinco anos } \\
\text { com déficit estatural para } \\
\text { idade }\end{array}$ & $\begin{array}{l}\text { Número de crianças menores de cinco anos com } \\
\text { déficit estatural atendidas na atenção básica x } \\
100 \text { / número total de crianças atendidas na } \\
\text { atenção básica no ano de referência. }\end{array}$ & $\begin{array}{l}\text { Quantitativa } \\
\text { continua }\end{array}$ & Sisvan web \\
\hline \multicolumn{4}{|l|}{$\begin{array}{l}\text { Variável independente } \\
\text { principal }\end{array}$} \\
\hline $\begin{array}{l}\text { Percentual de compra } \\
\text { direta de alimentos da } \\
\text { agricultura familiar para } \\
\text { O PNAE }\end{array}$ & $\begin{array}{l}\text { Valor em reais do recurso do PNAE utilizado para } \\
\text { compra de alimentos da agricultura familiar X } \\
100 \text { / valor total em reais do recurso do PNAE } \\
\text { repassado para o município no ano de referência. }\end{array}$ & $\begin{array}{l}\text { Quantitativa } \\
\text { contínua }\end{array}$ & FNDE \\
\hline \multicolumn{4}{|l|}{$\begin{array}{l}\text { Variáveis independentes } \\
\text { - contextuais }\end{array}$} \\
\hline $\begin{array}{l}\text { Taxa de mortalidade } \\
\text { infantil }\end{array}$ & $\begin{array}{l}\text { Número de crianças que não deverão sobreviver } \\
\text { ao primeiro ano de vida em cada } 1.000 \text { crianças } \\
\text { nascidas vivas. }\end{array}$ & $\begin{array}{l}\text { Quantitativa } \\
\text { contínua }\end{array}$ & PNUD \\
\hline Indice de Gini & $\begin{array}{l}\text { Mede o grau de desigualdade existente na } \\
\text { distribuição de indivíduos segundo a renda } \\
\text { domiciliar per capita. Seu valor varia de 0, quando } \\
\text { não há desigualdade (a renda domiciliar per } \\
\text { capita de todos os indivíduos tem o mesmo valor), } \\
\text { a 1, quando a desigualdade é máxima (apenas } \\
\text { um indivíduo detém toda a renda). O universo } \\
\text { de indivíduos é limitado àqueles que vivem em } \\
\text { domicílios particulares permanentes. }\end{array}$ & $\begin{array}{l}\text { Quantitativa } \\
\text { contínua }\end{array}$ & PNUD \\
\hline $\begin{array}{l}\text { \% dos ocupados no setor } \\
\text { agropecuário - } 18 \text { anos } \\
\text { ou mais } 2010\end{array}$ & $\begin{array}{l}\text { Razão entre o número de pessoas de } 18 \text { anos ou } \\
\text { mais ocupadas no setor agropecuário e o número } \\
\text { total de pessoas ocupadas nessa faixa etária. }\end{array}$ & $\begin{array}{l}\text { Quantitativa } \\
\text { contínua }\end{array}$ & PNUD \\
\hline $\begin{array}{l}\text { \% da população em } \\
\text { domicílios com água } \\
\text { encanada } 2010\end{array}$ & $\begin{array}{l}\text { Razão entre a população que vive em domicílios } \\
\text { particulares permanentes com água canalizada } \\
\text { para um ou mais cômodos e a população total } \\
\text { residente em domicílios particulares permanentes } \\
\text { multiplicado por } 100 \text {. A água pode ser proveniente } \\
\text { de rede geral, de poço, de nascente ou de } \\
\text { reservatório abastecido por água das chuvas ou } \\
\text { carro-pipa. }\end{array}$ & $\begin{array}{l}\text { Quantitativa } \\
\text { contínua }\end{array}$ & PNUD \\
\hline $\begin{array}{l}\text { \% da população em } \\
\text { domicílios com coleta de } \\
\text { lixo } 2010\end{array}$ & $\begin{array}{l}\text { Razão entre a população que vive em domicílios } \\
\text { com coleta de lixo e a população total residente } \\
\text { em domicílios particulares permanentes } \\
\text { multiplicado por } 100 \text {. Estão incluídas as situações } \\
\text { em que a coleta de lixo é realizada diretamente } \\
\text { por empresa pública ou privada, ou o lixo é } \\
\text { depositado em caçamba, tanque ou depósito fora } \\
\text { do domicílio, para posterior coleta pela prestadora } \\
\text { do serviço. São considerados apenas os domicílios } \\
\text { particulares permanentes localizados em área } \\
\text { urbana. }\end{array}$ & $\begin{array}{l}\text { Quantitativa } \\
\text { contínua }\end{array}$ & PNUD \\
\hline $\begin{array}{l}\text { \% de crianças de } 0 \text { a } 5 \\
\text { anos fora da escola } 2010\end{array}$ & $\begin{array}{l}\text { Razão entre o número de crianças de } 0 \text { a } 5 \text { anos } \\
\text { que não frequentam a escola e o total de crianças } \\
\text { nesta faixa etária multiplicado por } 100 .\end{array}$ & $\begin{array}{l}\text { Quantitativa } \\
\text { contínua }\end{array}$ & PNUD \\
\hline $\begin{array}{l}\text { IDHM - Índice de } \\
\text { Desenvolvimento } \\
\text { Humano Municipal } 2010 \\
\end{array}$ & $\begin{array}{l}\text { Média geométrica dos índices das dimensões } \\
\text { renda, educação e longevidade, com pesos iguais. }\end{array}$ & $\begin{array}{l}\text { Quantitativa } \\
\text { contínua }\end{array}$ & PNUD \\
\hline
\end{tabular}


(Continuação)

\begin{tabular}{|c|c|c|c|}
\hline Variável & Descrição & Tipo & $\begin{array}{c}\text { Fonte de } \\
\text { dados }\end{array}$ \\
\hline $\begin{array}{l}\text { Taxa de analfabetismo - } \\
15 \text { anos ou mais } 2010\end{array}$ & $\begin{array}{l}\text { Razão entre a população de } 15 \text { anos ou mais de } \\
\text { idade que não sabe ler nem escrever um bilhete } \\
\text { simples e o total de pessoas nesta faixa etária } \\
\text { multiplicado por } 100 \text {. }\end{array}$ & $\begin{array}{l}\text { Quantitativa } \\
\text { contínua }\end{array}$ & PNUD \\
\hline $\begin{array}{l}\text { Expectativa de anos de } \\
\text { estudo aos } 18 \text { anos de } \\
\text { idade em } 2010\end{array}$ & $\begin{array}{l}\text { Número médio de anos de estudo que uma } \\
\text { geração de crianças que ingressam na escola } \\
\text { deverá completar ao atingir } 18 \text { anos de idade, se } \\
\text { os padrões atuais se mantiverem ao longo de sua } \\
\text { vida escolar. }\end{array}$ & $\begin{array}{l}\text { Quantitativa } \\
\text { contínua }\end{array}$ & PNUD \\
\hline Renda per capita & $\begin{array}{l}\text { Razão entre o somatório da renda de todos os } \\
\text { indivíduos residentes em domicílios particulares } \\
\text { permanentes e o número total desses indivíduos. } \\
\text { Valores em reais de } 1^{\circ} \text { de agosto de } 2010 \text {. }\end{array}$ & $\begin{array}{l}\text { Quantitativa } \\
\text { contínua }\end{array}$ & PNUD \\
\hline $\begin{array}{l}\text { Proporção de crianças } \\
\text { extremamente pobres }\end{array}$ & $\begin{array}{l}\text { Proporção dos indivíduos com até } 14 \text { anos de } \\
\text { idade que têm renda domiciliar per capita igual ou } \\
\text { inferior a R\$ } 70,00 \text { mensais, em reais de agosto } \\
\text { de } 2010.0 \text { universo de indivíduos é limitado } \\
\text { àqueles que vivem em domicílios particulares } \\
\text { permanentes. }\end{array}$ & $\begin{array}{l}\text { Quantitativa } \\
\text { contínua }\end{array}$ & PNUD \\
\hline $\begin{array}{l}\text { Taxa de desocupação - } \\
\text { dez anos ou mais }\end{array}$ & $\begin{array}{l}\text { Percentual da população economicamente ativa } \\
\text { (PEA) nessa faixa etária que estava desocupada, } \\
\text { ou seja, que não estava ocupada na semana } \\
\text { anterior à data do Censo, mas havia procurado } \\
\text { trabalho ao longo do mês anterior à data dessa } \\
\text { pesquisa. }\end{array}$ & $\begin{array}{l}\text { Quantitativa } \\
\text { contínua }\end{array}$ & PNUD \\
\hline $\begin{array}{l}\text { Despesas com agricultura } \\
\text { per capita - municipal } \\
\text { (em reais) }\end{array}$ & $\begin{array}{l}\text { Razão entre a média das despesas municipais com } \\
\text { agricultura familiar dos municípios brasileiros no } \\
\text { período de } 2013 \text { a } 2017 \text { e a população total do } \\
\text { município em } 2010 \text {. }\end{array}$ & $\begin{array}{l}\text { Quantitativa } \\
\text { contínua }\end{array}$ & Ipea e IBGE \\
\hline
\end{tabular}

